\title{
คteknokultura
}

\#Rodriguez PRIETo, R. (2016). Internet como oportunidad y problema en los movimientos sociales contemporáneos , Revista Teknokultura Vol. 13(1), 289-307.

Recibido: 18-02-2016

Aceptado: 15-04-2016
Open peer review

http://revistas.ucm.es/index.php/TEKN/pages/view/opr-51930

\section{Internet como oportunidad y problema en los movimientos sociales contemporáneos}

\author{
The Internet as opportunity and challenge \\ in contemporary social movements \\ Rafael Rodríguez Prieto \\ Universidad Pablo de Olavide \\ rrodpri@upo.es
}

\section{RESUMEN}

Este artículo explora la relación entre el surgimiento de "nuevos" movimientos sociales (el $15 \mathrm{M}$ y Occupy) e Internet. Los nuevos medios sociales permiten a estos movimientos sociales desplegar una presencia significativa tanto en espacios cibernéticos como físicos. Su futuro estará caracterizado por movinets en tanto formas de activismo más eficiente. Su mayor capacidad de hacer circular ideas y propuestas alterará inevitablemente las dinámicas de la movilización social.

\section{Palabras Clave}

Movilización; movimientos sociales; Internet; participación; 15M; Occupy. 


\section{Abstract}

This paper explores the relationship between the rise of "new" social movements (15-M and Occupy) and the Internet. The new social media gives rise to new kinds of social movements which embed this technology from the moment of conception. The future of social movements will be characterised by movinets, which will have the effect of developing new efficient ways of activism. The movinets, with their embedded technology and capacity to circulate ideas among different spheres of reality, have a potential to alter the dynamics of social mobilisation.

\section{KEYWORDS}

Mobilization; social movements; Internet; participation; 15M; Occupy.

\section{Contenidos}

1. Introducción

2. Una anatomía del $15 \mathrm{M}$ : proceso histórico y discurso

3. Movinet: movilización política e Internet Oportunidades y límites de la Red

4. Conclusiones

5. Referencias

\section{Contents}

1. Introduction

2. An Anatomy of $15 \mathrm{M}$ : historical process and discourse

3. Movinet: political mobilization and the Internet Challenges and constraints in the Internet

4. Conclusions

5. References 


\section{Introducción}

El 15 de mayo de 2011 España vivó una manifestación que concitó el apoyo y la simpatía de amplios sectores sociales. Por primera vez, en la historia reciente, emergía un movimiento de protesta cuyo fin era realizar transformaciones de calado del modelo político y socioeconómico. Al contrario que en otras ocasiones -antiglobalización- el 15M contaba con un masivo apoyo ciudadano.

\section{Una anatomía del 15M: proceso histórico y discurso}

En 2016 estos movimientos parecen haber perdido una buena parte del impulso con el que nacieron. Del 15M nos queda su legado. La generación de un nuevo sentido común surgido del acontecimiento y del proceso de politización masiva del 15M (Toret, 2015). Algunos colectivos políticos intentaron tomar el testigo y afirmarse como sus herederos con desigual fortuna (Podemos o Partido X). Otros grupos, antiguos o nuevos, de resistencia sectorial frente a medidas económicas neoliberales como la PAH (Plataforma de Afectados por la Hipoteca) o las "mareas ciudadanas" contra la privatización de la sanidad o la educación, han tratado de mantener algunas de las líneas de actuación señeras de la movilización y de vincularla con la crítica sistémica del modelo económico y político hegemónico. El colectivo que dio lugar al 15M -Democracia Real Ya- continúa, pero su actividad se centra en apoyar estas movilizaciones. Su influencia ha decaído enormemente. Peor suerte ha tenido el movimiento Occupy, en el que, como señala Castañeda, el 15M tuvo una relevante influencia (Castañeda, 2012, p. 316). Occupy continúa actuando como tal, aunque con escasa visibilidad. Sus acciones pusieron de relieve la influencia del sector financiero en la política (el gobierno de Wall Street). Hoy, sus ideas y propuestas cuentan con escaso eco en la opinión pública. Algunos de sus activistas han terminado por apoyar a Sandersen la carrera por la candidatura Demócrata. Desde sus inicios, cultivaron la desobediencia política, algo bastante novedoso en EE.UU. El movimiento realizó actividades pacíficas de protesta. Sigue contando con el apoyo der un nutrido grupo de intelectuales que lo respaldan desde la universidad. No obstante, este respaldo es escaso comparado con el que realizan los think tanks neoliberales a grupos de derecha. En el caso de México y su movimiento yo soy 132, su empuje se desvaneció tras las sospechas de fraude masivo de las elecciones de 2012 que situaron a Peña Nieto-PRI- al frente del gobierno y las protestas por desapariciones de estudiantes que aún permanecen sin aclarar. 
La hipótesis planteada por este trabajo es que estos colectivos se corresponden con una nueva clase de movimiento social denominada movinets, que agruparía tanto al $15 \mathrm{M}$ como a movimientos similares (Hadzelek y Rodríguez, 2014), que mantiene una relación constante con la Red, con sus oportunidades, problemas y en sus propuestas. Movinets puede definirse como una categoría que incluye colectivos sociales desarrollados en la última década y que constituyen un paso más en el proceso de aprendizaje de los movimientos sociales y la clase trabajadora o precarizada que los compone de forma mayoritaria. Estos colectivos han insertado las tecnologías de la información en su acción hasta el punto de que son inseparables de las mismas; una simbiosis tecnopolítica que incrementa sus oportunidades y los expone también a riesgos. Por último, los movinets son movimientos sociales que asumen un enfoque relacional en sus causas en vez de particularista. Esto significa que pueden tener objetivos amplios en su actividad, en vez de una reivindicación muy específica y, de existir la misma, este tipo de acción colectiva sectorial se encuentra relacionada con otros problemas sociales (como la plataforma antidesahucios que vincula sus actuaciones con un sistema financiero pensado para beneficiar a los grandes capitales, con la complicidad de élites políticas). En definitiva los movinets reincorporan e reintroducen la cuestión de clase, que ha sido matizada u olvidada en los nuevos movimientos sociales, como fruto de un aprendizaje que partió del movimiento antiglobalización de los 90, encontrando en las nuevas tecnologías de la información la vía para el desarrollo de su actividad.

Internet ha tenido una gran influencia en la generación y desarrollo de estos movimientos y forma parte del ADN de los mismos. No es posible comprenderlos sin Internet y por ello supone una innovación respecto a los movimientos tradicionales o clásicos -sindicatos, partidos- y los nuevos movimientos sociales, de finales del pasado siglo, más centrados en elementos de carácter identitario y ajenos a esta tecnología. Esta relación debe ser asumida de manera crítica y responsable.

No obstante, cabe advertir que los movinets, al contrario que los nuevos movimientos sociales, cuentan con alternativas al modelo socioeconómico hegemónico. Se trata de una diferencia significativa. La necesidad de transformaciones significativas de las relaciones sociales, políticas y de producción emerge de manera evidente en sus programas y reivindicaciones. También, probablemente, porque los nuevos movimientos sociales se desarrollaron en un contexto donde el estado del bienestar estaba muy presente. El abandono de esta idea en las últimas décadas puede explicar esta característica heredada del movimiento antiglobalización y el Foro Social Mundial (FSM). También cabe añadir las grandes transformaciones tecnológicas de los últimos lustros y su impacto, al menos, en los trabajadores en occidente. 
La fusión entre movimiento social que cuestiona el modelo político y socioeconómico e Internet no es sencilla. Por un lado, tenemos la mitología que rodea a la Red y la sublimación tecnológica a la que no han sido ajenos otros medios como la televisión o el teléfono (Mosco, 2004). Por otro, los desafíos relacionados con el acceso. Como cualquier otra tecnología, Internet debe ser objeto de una reflexión concienzuda que abandone posiciones acríticas. El mito digital permanece inalterado junto con una falta de cuestionamiento de los canales privados/mercantiles que se usan en las comunicaciones -como Facebook o Twitter. Se pasa por alto que Internet no es una plaza pública como algunos gobiernos y corporaciones pretenden hacer creer, sino que está condicionada por un cuerpo físico, sometido a unas jurisdicciones y controles a la vez que a unas corporaciones privadas con afán de lucro y posiciones prácticamente monopolísticas. Mito y acceso son cuestiones muy relevantes en el análisis.

El acceso a Internet es decisivo. De acuerdo a la Unión Internacional de las Telecomunicaciones (UIT, 2015), un 43,3 \% de la población mundial, unos 3.200 millones de personas, cuentan con acceso a Internet. Esta cifra debe ser matizada por las diferencias geográficas existentes en la velocidad de conexión o capacidad de descarga, según el informe Medición de la Sociedad de la Información de la UIT. De acuerdo a esta organización, en los países desarrollados, el 81,3\% de los hogares disponen ahora de acceso a Internet, mientras que solo el 34,1 \% de los hogares en los países en desarrollo y el 6,7\% de los 48 países con menor renta. Sin embargo, el número de usuarios de Internet en los países en desarrollo se ha duplicado en los últimos cinco años (2010-2015). En esta parte del mundo habitan dos tercios de las personas conectadas a Internet. La banda ancha móvil ha crecido exponencialmente en número de suscripciones en todo el mundo; en cinco años, se ha pasado de 800 millones en 2010 a 3.500 millones en 2015 (EFE, 2015). Un hecho como este condiciona el desarrollo de los movinets por regiones diferentes a Europa, EE.UU., Japón o Australia, si bien es cierto que las propias condiciones de desigualdad hacen que buena parte de la población del planeta vea limitada su jornada diaria a tratar de lograr subsistir. La radicalización de la alienación, paralela al proceso de mercantilización, implica una dificultad añadida para estos colectivos. En este contexto, el Secretario General de Naciones Unidas estableció el Foro para la Gobernanza de Internet (FGI) donde se incluyen gobiernos, sector privado, sociedad civil y comunidad técnica y académica. Desde 2006 hasta el momento se han celebrado reuniones anuales. La falta de avances significativos en la regulación o en la concentración del negocio de Internet en pocas manos es evidente. Todavía más: el creciente espionaje y uso sin control de datos personales son muestra fehaciente de sus pobres resultados. 
Internet puede ser una herramienta para la emancipación, pero también para un control sin límites. Cualquier oposición debe partir de un reconocimiento de una vigilancia global y sus efectos en la vida diaria (Lee, 2014). Tanto troyanos introducidos por gobiernos para controlar a ciudadanos como perfiles de gustos y preferencias que crean las empresas, apropiándose de forma gratuita de información muy valiosa para ellos, serían manifestaciones de una tecnología al servicio de relaciones de dominio. Por un lado, el software espía representado por programas como PRISM, o Carnivore (o DCS1000) o ECHELON. Por otro, el datismo, como su expresión máxima, desarrolla no solo un rastreo de nuestras actividades diarias, sino que además permite una publicidad personalizada o la predicción del comportamiento futuro a partir de la huella digital (Segura, 2014). Este datismo revelaría patrones colectivos de comportamiento que escapan a la conciencia del individuo y apoderarse del comportamiento de las masas a un nivel que escapa de la conciencia (Han, 2014, p. 98). El refinamiento de los sistemas de control y vigilancia gracias a la Red es un factor que no puede olvidarse; tampoco las posibilidades de cortocircuitarlos para generar cambios reales.

Como señala Negri, la emancipación es práctica política efectiva de resistencia y creación cooperativa. No podemos entenderla en un sentido iluminista ni escatológico (Negri, 2012). Es por ello que no debe descuidarse una mirada crítica sobre la propia Red y los mitos que la envuelven. Algunos activistas del 15M, conscientes de estas amenazas, han desarrollado canales alternativos como Lorea.

\section{Movinet: movilización política e Internet Oportunidades y límites de la Red}

Los movimientos sociales contemporáneos, a los que denominados movinets, poseen una organización y composición sociológica que los identifica como colectivo de colectivos, es decir, dentro del mismo conviven diversos grupos que se identifican con un discurso único. En el caso del 15M con el "no nos representan" y en el de Occupy con Walll Street Government y somos el 99\% (Winner, 2014). Los movinets no surgen de la nada: reaccionaron contra una situación que consideraron injusta y tomaron las herramientas tecnológicas con las que estaban familiarizados. En esencia, las movilizaciones son medios productivos que canalizan el descontento (Time, 2011, p. 28). Desde un punto de vista cualitativo, la burbuja generada por el afán especulativo del capital financiero y sus efectos desembocó en un clima de descontento social, agravado por las consecuencias de tres décadas de políticas neoliberales sustentadas en el llamado "Consenso de Washington" y por la evidente descom- 
posición de un modelo representativo al que se le han amputado de forma creciente las políticas realmente relevantes (monetaria, de regulación del mercado, por ejemplo) y controlado por la deuda generada, en gran medida, por el salvamento del sistema financiero y por la descapitalización del sector público mediante la entrada, con consecuencias ruinosas, del sector privado en la gestión de servicios básicos (por ejemplo, la muy discutible "colaboración" público-privada en el sector sanitario). Por un lado, se empobrecía a los ya perjudicados por la misma y, por otro, se acrecentaron las desigualdades sociales. Así, desde una perspectiva cuantitativa, tal y como se ha preocupado de insistir en gran parte de su trabajo Navarro, desde comienzos de los ochenta, el crecimiento de las rentas superiores, cifrado en un 5\% superior de la población, se aceleró respecto al del resto de la población. Lo que supuso que el $40 \%$ (los dos quintiles inferiores de la población) apenas vio crecer sus ingresos durante el periodo 1979-2007 (un promedio del 0,2\%). Durante la crisis (20072015) se recrudeció el descenso en sus ingresos $-2,4 \%$. La transferencia de rentas del trabajo al capital es un hecho incontestable. Así, mientras en el periodo 1948-1973 el crecimiento de los salarios fue de un $91,3 \%$ de crecimiento acumulado, semejante al de la productividad $-96,7 \%$-, en el periodo 1973-2014 el crecimiento de los salarios fue de un 9,2\%, empero el de la productividad fue del 72,2\% (Navarro, 2016).

Son éstas -el crecimiento de la brecha entre ricos y trabajadores- el contexto donde se produce en Europa y EE.UU. la emergencia de movimientos como el 15M (Tejerina, et al., 2013, pp. 381, 384) u Occupy. Los procesos sociales e históricos que actúan contra una situación percibida como injusta es lo que aúna a distintos colectivos bajo unos mínimos que están relacionados con aquello a lo que se reacciona. No son la suma de una pluralidad de ellos, sino colectivos diversos que se sitúan bajo un mínimo común denominador (Candón, 2013). Se apreció en las movilizaciones del 15M y hoy en día, en las confluencias dadas a nivel municipal. No existen elementos suficientes para afirmar que la fragmentación en grupos heterogéneos no apareciera o pudiera percibirse como algo subyacente, pero sí se puede señalar que fue desplazada por la presentación de un bloque cohesionado alrededor de una serie de mensajes.

Los movinets cuestionan tanto el elitismo de la política como la propia idea de representación. En el caso del 15M se denunció con el lema de "no nos representan", que estas estructuras de representación han dado paso a un modelo elitista de poliarquía electoral (García Espín, 2012). En el caso de Occupy se usó la consigna de "somos el 99\%". Esta idea es sobre todo política, ya que con ella se trata de trasladar a la opinión pública que los intereses del 99\% no son representados en las instituciones de gobierno (Taylor et al., 2011, pp. 16-17). Los movinets denuncian el poder creciente de organizaciones que no han sido elegidas por nadie y su influencia tanto en las decisiones como en las no-decisiones que 
adoptan los gobiernos. Tal y como ha sido estudiado por una parte relevante de la filosofía política, el ideal de la representación se compadece más con el ideal elitista del gobierno representativo concebido por el liberalismo, que con una dificultad de contar con la opinión de los ciudadanos, (Arblaster, 1993). El individualismo propietario y capitalista condicionó la representación de sus inicios a la exclusión de los no propietarios -también, no blancos o no hombre- y para ello se legitimó con los ideales kantianos de imparcialidad y objetividad (Young, 1990, p. 93). De hecho, el propio proceso participativo que jalona la democracia capitalista se ha fundamentado en exclusión de los no propietarios y una oposición entre mediación e inmanencia que hace continúa abstracción de las condiciones materiales de los sujetos (Negri, 1993). Solo después de la II Guerra Mundial y con el estado del bienestar, se posibilitó una ampliación de la participación que ha quedado de nuevo limitada por la contrarrevolución neoliberal de los ochenta (Roper, 2013, p. 203). Ello ha generado un proceso de traslación de la decisión hacia organizaciones de integración regional, donde los parlamentos estatales tienen cada vez menos poder y capacidad de influencia, organizaciones multilaterales y a tratados de "libre comercio" realizados al dictado de las grandes corporaciones privadas. El poder desmedido de las entidades financieras se demuestra cada día en las bolsas de todo el mundo y en la sangría de los ingresos estatales, gracias a la ingeniera financiera que carga sobre los trabajadores, de forma prácticamente exclusiva, el pago de impuestos que mantienen los servicios básicos. El "no nos representan" es la constatación material de una intuición que aún no ha adquirido el grado de certeza, pues de ser así, el modelo representativo hegemónico habría quedado totalmente deslegitimado a ojos de la opinión pública, cosa que todavía, al menos de forma clara y patente, no ha sucedido. Incluso se podría añadir, que algunas organizaciones políticas que se reclaman herederas del $15 \mathrm{M}$ han contribuido a fortalecer la legitimación de un modelo representativo fuertemente intervenido desde instancias ajenas a la ciudadanía. Las apelaciones a la nueva política han de ser tomadas con cautela. No ha transcurrido demasiado tiempo, pero ya se aprecian comportamientos organizativos escasamente tributarios del $15 \mathrm{M}$. Todo ello contrasta sobremanera con la propia lógica organizativa del 15M. Soriano (2014) ha descrito interesantes vías para la mejora de las instituciones representativas, del sistema electoral (Alarcón y Soriano, 2004) o, gracias a Internet, el propio $15 \mathrm{M}$ ha diseñado mecanismos tecnopolíticos capaces de reducir la influencia de la representación -des-representación- como la propuesta de Democracia 4.0 (Jurado, 2014).

Desde el punto de vista teórico los movinets son potencialmente movimientos de desobediencia política. Este elemento es trascendental si la comparamos con la desobediencia civil, practicado por colectivos con los de defensa de los Derechos Civiles en EE.UU. La 
desobediencia civil acepta la legitimidad de la estructura política y de las instituciones que se derivan de ella, pero resisten la autoridad moral del derecho resultante de las mismas. La desobediencia civil acepta la condena consecuencia de tal desobediencia y cuestiona la justicia de las normas que se deriva de una estructura política que sí es aceptada. En cambio la resistencia política cuestiona la propia estructura política. Las demandas del $15 \mathrm{M}$ de cambios profundos en la relaciones de poder y en la estructura política y en el gobierno estarían más cercanas a una desobediencia política que civil. En el caso de Occupy es clara la crítica total a un modelo que los activistas consideran que ha sido usurpado y modelado según los intereses de Wall Street (Harcourt, 2013, pp. 46-47).

Los movinets integran Internet en su ADN. No es una característica o dimensión más de su actuación, sino que forma parte de la propia concepción del movimiento que integra la red como parte ontológica del mismo. Otros movimientos sociales anteriores han incorporado Internet en su actuación, pero en ellos Internet es un vehículo más de difusión que a veces es hasta desplazado por otros más tradicionales. Los movinets no entienden la movilización sin la Red. Como se señaló anteriormente, esta característica central no es pacífica. Reviste consecuencias positivas y negativas y debe ser evaluada con cuidado. En los propios activistas se detecta un optimismo tecnológico que puede resultar peligroso.

El caso de Snowden -el ex agente de la CIA que reveló el control al que EE.UU. somete las comunicaciones a través de Internet- nos ha desvelado un uso indebido y preocupante de Internet. El hecho de que las ventas de la novela de Orwell 1984 subieran un $7000 \%$ durante el verano de 2013 representa con claridad el impacto de estos descubrimientos. Quizá la opinión pública nunca fue completamente ingenua en lo que respecta a un mal uso de la Red, pero nunca se había presentado de forma tan cruda dos realidades que convergen en el caso Snowden: por un lado, el control estatal y, por otro, la complicidad de las grandes empresas de Internet. Se supone que estas corporaciones debían velar por la privacidad y los intereses de sus clientes y, sin embargo, no estaban haciéndolo. Es necesario llevar a cabo una evaluación seria de Internet como instrumento contra el autoritarismo. El caso de Irán es fundamental, a lo que se podría añadir algunas de las frustrantes consecuencias de la denominada, con ciertas dosis de ingenuidad y tal vez precipitación por algunos, Primavera Árabe (Nair, 2011). Es necesario cuestionar tanto el ciberutopismo, como la centralidad de Internet.

Internet puede aumentar el poder de regímenes autoritarios (Morozov, 2011, pp. 27, 30 y 313) y a la vez ser un vehículo que fortalezca el control de las grandes corporaciones privadas sobre las personas (McChesney, 2013). Internet se presenta como un centro neurálgico de una actividad política que cuestiona la correlación de fuerzas políticas y so- 
cioeconómicas. Pero aunque fue clave en la dinámica del movimiento, no hubiera alcanzado el grado de impacto social que tuvo sin su presencia física en las plazas de España, como en Madrid fue la Puerta del Sol o en Nueva York con la toma simbólica de diversas zonas de la ciudad. En este sentido, el 15M y otros movimientos como Occupy entroncan con las ocupaciones históricas realizadas por trabajadores como protesta por sus condiciones laborales (Sherry, 2010). Los movinets se han beneficiado de un aprendizaje histórico de las olas de movilización habidas durante la historia; pero al mismo tiempo han sabido desarrollar mecanismos estratégicos propios para impulsar su acción, difundir su discurso e incrementar su base social. La lógica que se está imprimiendo a la Red, basada en una instantaneidad que repudia la memoria y la reflexión, no debiera evitar que estos colectivos se reconocieran en un pasado de lucha de clases y resistencias. Especialmente porque los movinets usan la Red como vehículo de deliberación y reflexión, pero también de organización tanto a mediano y largo plazo como de manera inmediata. No se desligan de la realidad y de los espacios físicos y, en consecuencia, tampoco debería hacerlo de la historia y los procesos aparejados a la misma.

La difusión que se consigue gracias a la Red es uno de los activos principales de los movinets. El papel de Internet es básico para entender el $15 \mathrm{M}$. Se trata de un instrumento decisivo para la ocupación del espacio público mediante el uso de una comunicación alternativa y de redes sociales como Twitter (ser trending topic), lo que redujo su dependencia de los medios tradicionales (Fuster Morell, 2012, p. 390). Estos elementos distintivos conviven con un cierto fetichismo tecnológico como herramienta de transformación social. Internet es una herramienta útil para organizar la movilización y el debate. La cultura de la conversación y del intercambio de información ha recibido un notable impulso gracias a la Red (Drew, 2013, p. 187), que han sido completadas por campañas dirigidas a conseguir un objetivo muy concreto (Maratea, 2014, p. 119). Las protestas del movimiento estudiantil de Gran Bretaña de principios del siglo XXI lograron reconducir el debate político de tal forma que se reconociera la relevancia del mismo movimiento (Linton, 2015, p. 204). Hay activistas que incluso afirman que con internet el desarrollo de la democracia será imparable (miembro de $15 \mathrm{M}$ ). Todo ello conforma una imagen amable de la Red en relación a los movimientos sociales contemporáneos. No obstante, este discurso ha de ser matizado y tomado con cautela.

La mercantilización de Internet supone un grave menoscabo en las posibilidades que ofrecen movimientos como el del 15M. Este límite es marcado por la presunción de neutralidad tecnológica y por un optimismo que opera como mecanismo que eclipsa las relaciones de poder inherentes a cualquier herramienta de comunicación (Rodríguez y Martínez, 2016). 
Las carencias regulatorias, la concentración de las grandes empresas relacionadas con Internet y la propia dinámica del neoliberalismo obligan a los movimientos sociales de nuestro siglo a llevar a cabo una seria y rigurosa reevaluación de las expectativas en torno a Internet. Por mucho que se insista, Internet no ha podido transformar la creciente mercantilización de la vida y de los recursos. ¿Cómo conciliar las expectativas de emancipación que despierta la nueva tecnología con un panorama de creciente desigualdad?

Junto con la necesaria cautela o salvaguarda cabe señalar que las posibilidades que Internet ofrece son de gran interés. El propio $15 \mathrm{M}$ a través de sus activistas se ha relacionado con proyectos como Guifi.net (una fundación que despliega una red de telecomunicaciones independiente de intereses mercantiles, mayoritariamente inalámbrica, con más de 32.900 nodos) que son plenamente conscientes de los desafíos de Internet y plantean vías de solución a los mismos de forma muy eficaz y audaz. Esto último es de singular importancia para entender el $15 \mathrm{M}$ como un nuevo movimiento social al que podemos calificar como movinet. El $15 \mathrm{M}$ y sus similares, a diferencia de movimientos anteriores, cuenta con Internet desde el principio -con sus límites y posibilidades. La Red se establece como un cauce decisivo de su acción política. Sus activistas se han aculturado en su uso.

El otro elemento relevante es el de la propuesta. Quizá la iniciativa señera en este sentido de un movinets fue la realizada por el 15M y denominada Democracia 4.0. De acuerdo a Francisco Jurado y Juan Moreno, creadores de la iniciativa, la propuesta consistía en la posibilidad de que cada uno de los alrededor de los 35 millones de ciudadanos españoles con derecho a voto pudiera recuperar su parte de soberanía y utilizarla en votaciones que sobre políticas concretas se llevan a cabo en el Congreso de los Diputados y hacerlo mediante la Red. Se dividiría 1 por el número de electores del censo (1/35.000000). El resultado sería la parte de soberanía de cada ciudadano. Cien mil personas equivalen a un escaño y así sucesivamente (1.000000 equivaldría a 100.000 electores). La consecuencia no sería que habría diputados que perdieran su voto, sino que se reduciría de forma ponderada el voto del conjunto de los disputados en función de su representación. Además éstos participarían en función del interés que tuvieran en la norma que se votara y los partidos políticos y representantes continuarían trabajando en el parlamento. La propuesta se ampara en la propia Constitución, así como en normas de parlamentos autonómicos que permiten el voto de diputados que se encuentren de baja mediante Internet

(https://15mpedia.org/wiki/Democracia_4.0). 
La propuesta Democracia 4.0 pretende cortocircuitar tanto la elitización radical de la política como la influencia de los grandes capitales financieros y empresariales. La votación sobre políticas, en vez de sobre élites, posibilita recomponer el consenso en torno a las instituciones representativas, al mismo tiempo que legitima decisiones que el legislativo debe tomar en torno a temas fundamentales para el conjunto de la ciudadanía.

La influencia económica de grandes empresas permanecería, pero las instituciones democráticas serían inmunes a las mismas, lo que redundaría en una bajada en los índices de corrupción y una mejora de los controles ciudadanos. La puerta giratoria no tendría los efectos perversos que en la actualidad posee, ya que se limitaría la posibilidad de que las empresas pretendieran influir en los reguladores de la actividad fichando a políticos para sus consejos de administración o presionando para que empleados suyos ocupen cargos de responsabilidad. Al ser más difícil poner en práctica ese tipo de influencia, a causa del voto que propone Democracia 4.0 y se desincentivaría.

¿Qué riesgos podría entrañar una experiencia? Las principales críticas que se podrían hacer a una propuesta como Democracia 4.0 provienen del aspecto tecnopolítico de la idea. Se pueden identificar dos amenazas evidentes:

(i) la seguridad del voto

(ii) los procesos privatizadores en el seno de Internet.

El primero de los problemas que plantearía un mecanismo teconopolítico como el estudiado implicaría extremar los controles de seguridad de los certificados electrónicos que permitirían el voto. Es evidente que el fraude también puede ser cometido con papeletas como sucedió en las elecciones de 2000 a la presidencia de EE.UU. en Florida. Pero no cabe duda de que la seguridad de tarjetas e incluso DNI electrónicos puede ser subvertida por expertos informáticos que en una votación de un proyecto legislativo de especial trascendencia podría actuar, bien por iniciativa propia, o a sueldo de un grupo que pretendiera falsificar el resultado final de la votación o bloquearla.

La segunda de las críticas de las que puede ser objeto esta idea es de mayor calado. Éstas cuestionarían el cauce, la vía que sería usada para poner en marcha el voto de políticas al que se denomina Democracia 4.0. Existe una notable ingenuidad en la manera en que se percibe Internet por buena parte de la opinión pública. Da la impresión de que no se tuviera en cuenta que Internet necesita para su funcionamiento toda una infraestructura física que se sitúa en lugares concretos y bajo jurisdicciones particulares. Las posibilidades que ofrece la tecnología nos sitúan en un entramado de relaciones de poder construidas mediante el cono- 
cimiento, que no podemos obviar. Tampoco deberíamos ignorar la trascendencia que tiene que estas relaciones de poder se puedan transformar en relaciones de dominación (Rodríguez y Martínez, 2016).

La consecuencia es que los suministradores de servicio podrían tener un papel muy relevante en la votación, así como las empresas propietarias de las redes y servidores por dónde discurriría la información que se usaría para llevar a cabo el proceso. Aunque nos dotásemos de software confiable y garantizáramos la transparencia en la comunicación, el grado de privatización y mercantilización de Internet podría tener un peso decisivo en la implantación y el desarrollo de la experiencia. Quizá, como señala Subirats, una solución exclusivamente técnica como la Red no baste para la resolución de desafíos tan complejos como la profundización en la democracia (Subirats, 2015), o desde una perspectiva más realista, su mero salvamento.

No es posible escindir Internet del contexto político y los procesos en que se desarrolla. Es cierto que Internet puede ayudar a la participación ciudadana, pero si el ánimo de la concepción hegemónica de lo político es antagonista de dicha participación ciudadana fortalecida, Internet será un mero reflejo de ello. Corremos el riesgo de confundir una elevada actividad en el cibermundo de redes sociales que usan nuestros datos para obtener beneficios, con un cambio real en las condiciones de vida y participación política de los ciudadanos. Los cambios en el voto y en la democracia deben también estar vinculados a transformaciones de los procesos que construyen a diario Internet y el propio modelo de relaciones productivas. Es necesario impulsar procesos emancipadores en Internet en detrimento de procesos de mercantilización y privatización radical de la misma. Junto con ello sería fundamental ampliar el impacto de iniciativas como las de guifi.net dedicadas a la construcción de redes ciudadanas de acceso a Internet a fin de contar con una infraestructura común.

Las críticas a este mecanismo de participación no ponen en cuestión la necesidad de ampliar y concretar un derecho a voto sobre las políticas en vez de sobre élites (Rodríguez, 2005); ni lo deseable que sería profundizar en la independencia del poder político de las presiones económicas de las grandes corporaciones. Estas críticas nos alertan, no tanto de los defectos de la iniciativa en sí, sino de relaciones de dominio fuertemente ancladas en la sociedad dispuestas a limitar o cortocircuitar un despliegue de la misma. Y de esta manera, se pone de relevancia la necesidad de contextualizar Internet y las posibilidades que ofrece en la sociedad donde se sitúa y las relaciones de poder y dominio que interactúan con la Red. Si queremos más democracia, no solo debemos mejorar nuestras herramientas, sino 
ser más conscientes de que el perfeccionamiento de la misma depende de una serie de variables que se dejan en manos de operadores privados.

\section{Conclusiones.}

David Graeber señala que un año en términos de movimientos sociales es nada, ya que los movimientos que en EE.UU. han logrado sus objetivos, como el feminista o el abolicionista, han tardado décadas en conseguirlos; pero cuando se logran los resultados marcan una época (Graeber, 2013: 149). Es cierto que las transformaciones sociales llevan su tiempo. Sin embargo, los tiempos de los movimientos deben coordinarse con la propia aceleración que imprimen las sociedades a los acontecimientos y la certeza de que hay momentos cruciales para cristalizar los cambios. 15M y Occupy tuvieron un momento de auge y también de declive. El 15M ha representado un gran éxito colectivo, aunque solo fuera por concitar un gran apoyo popular y por el hecho de que los problemas reales de las personas ocuparan durante un tiempo la agenda política. Su legado, vinculado con dotar de prestigio a la acción colectiva y de mostrar la necesidad de alternativas es un fruto evidente del $15 \mathrm{M}$. Incluso en declive, movimientos sociales sectoriales que actualmente gozan de gran presencia y apoyo, han aprovechado su prestigio y la ola de simpatía que despertó. Caso de la Plataforma de Afectados por la Hipoteca -más clásico y preexistente- o \#15MpaRato, más influido por el uso de las redes sociales y consecuencia directa del 15M.

El contexto donde se han desarrollado los movinets no es fácil. El modelo representativo está en descomposición. El capitalismo es un modelo cultural, económico y político corrupto que ya no admite correcciones como las que estableció el estado del bienestar de postguerra. La traslación de la decisión a instancias incontrolables por los ciudadanos hace que nuestras democracias sean "pequeñas". Dicho empequeñecimiento viene motivado por esta carencia de control de las decisiones gubernamentales por parte de la ciudadanía. Las políticas realmente trascendentes han sido absorbidas por otros entes supranacionales. La deuda se ha constituido en un factor decisivo para el incremento del control. La reciente crisis que apunta a un fraude masivo utilizando paraísos fiscales no es nueva. Pero se trata de la evidencia de que las buenas palabras posteriores a la caída de Lehman Brothers, vinculadas con una peculiar "refundación del capitalismo" no eran nada, más que propaganda. La democracia pequeña implica que la ciudadanía podrá decidir, pero sobre cuestiones menores, que no afecten al aparato político y económico hegemónico. Todo ello en un contexto no solo de pérdida de derechos sociales, sino de derechos dados por sentado, como los civiles. La mercantilización extrema de Internet y su uso para el control de flujos de 
información y el espionaje masivo gracias a acuerdos entre los principales actores comerciales del sector $\mathrm{y}$ las agencias gubernamentales de inteligencia son los primeros responsables de una situación que tiende a ser preocupante. La inversión en la industria biotecnológica de estas grandes empresas muestra un futuro de control y desigualdad difícil de imaginar.

El principal reto de los movinets será quebrar este modelo de relaciones productivas, que gracias a los procesos de automatización, puede terminar por desplazar aún más a los seres humanos; hacerlos completamente prescindibles, muy al contrario de lo que había pensado el marxismo autonomista con la idea de trabajo vivo. Los movinets tienen la oportunidad de servirse de la tecnología para llevar a cabo las transformaciones del modelo social y productiva imprescindibles para la democracia. También de conectar Internet con la calle, tal y como nos dicta el legado del 15M y Occupy. Solo es necesario ser consciente de los límites de la Red y actuar de forma más creativa, resolutiva y activa.

Los movimientos sociales que integran Internet en su actuación desde su momento fundacional cuentan con unas dinámicas muy positivas que les permiten un alto grado de impacto casi dese el comienzo de su actuación. Este hecho justifica el uso de una nueva nomenclatura para los mismos, tal y como se ha defendido en este trabajo. Las diferentes redes sociales y las herramientas que brinda la Red les permiten organizar campañas y movilizaciones muy complicadas de llevar a cabo sin el concurso de esta tecnología. El impacto y eficacia de las mismas a medio y largo plazo es otra cuestión. La movilización que desarrollan los movimientos sociales parece colisionar con una realidad en la que los poderes neoliberales no ceden en sus procesos de colonización social. Al mismo tiempo, existen notables cautelas que los movinets deben tener en cuenta en relación a la apropiación del espacio cibernético por grandes empresas de Internet. Como se ha analizado, se trata de un espacio donde existe una alta concentración de proveedores de servicio y contenido, lo que pudiera dificultar la acción de colectivos realmente transformadores.

Los movinets pueden aprovechar la Red como un resorte tecnosocial primario dirigido a la activación de campañas que desarrollen la concienciación social. Esta vía precisará de otras herramientas y dinámicas si realmente se pretende consolidar la actividad y objetivos de estos colectivos. Internet por sí solo no es suficiente para el desarrollo de una estrategia a largo plazo. Quizá los movinets sean el inicio de un proceso más largo y continuado en el tiempo que posibilite un cambio real a escala planetaria. Aunque, tal vez, estos colectivos hayan demostrado más debilidades que fortalezas. Optar por la primera versión conduce a mantener la esperanza. La segunda implica pensar que es necesario desarrollar una acción 
colectiva con menos debilidades organizativas y que comprenda que Internet es tan sólo una herramienta, ya que al final el espacio físico resulta decisivo para el cambio. 


\section{Referencias}

Alarcón, C. y Soriano, R. L. (2004). Justicia Electoral: un nuevo modelo de elecciones para España. Córdoba: Almuzara.

Arblaster, A. (1993). Democracia. Madrid: Alianza.

CAndón, J. (2013). Toma la calle, toma las redes. El movimiento $15 M$ en Internet. Sevilla: Atrapasueños.

Castañeda, E. (2012). “The Indignados of Spain: A Precedent to Occupy Wall Street”. Social Movement Studies: Journal of Social, Cultural and Political Protest. 11:3-4.

EFE. (2015). “Tecnologías de la Información”, disponible en:

$<$ http://www.efe.com/efe/america/tecnologia/un-43-3-de-la-poblacion-mundial-tieneacceso-a-internet/20000036-2777245>, [Consultado el 11/04/16]

Fuster, M. (2012). “The Free Culture and 15M Movements in Spain: Composition, Social Networks and Synergies", Social Movement Studies: Journal of Social, Cultural and Political Protest, 11:3-4.

García, P. (2012). "El 15M: De vuelta al barrio como espacio político", Revista Internacional de Pensamiento Político, 7, pp. 291-310.

Graeber, D. (2013). The Democracy Project. A History. A crisis. A Movement. London: Allen Lane.

Harcourt, B. (2013). "Political Disobedience". En Mitchell, W. J. T., Harcourt, Bernard, Taussig, Michael (ed). Occupy. Three Inquiries in Disobedience. Chicago: The University of Chicago Press.

Hadzelek, A. y Rodríguez, R. (2014). "Movinets and the Future of Social Movements: How $15 \mathrm{M}$ and Occupy Revolutionised Political Protest”. En VV.AA., Essays 2014: Politics. Melbourne: Australian Scholarly Publishing.

Han, B. C. (2014). Psicopolítica. Barcelona: Herder.

Hughes, N. (2011). "Young People Took to the Streets and all of a Sudden all of the Political Parties Got Old': The 15M Movement in Spain”. Social Movement Studies, 4 Vol. 10.

JuRAdo, F. (2014). Nueva gramática política. De la revolución en las comunicaciones al cambio de paradigma. Barcelona: Icaria.

JuVENTUd SIN FUTURo (2011). Juventud sin futuro. Barcelona: Icaria. 
Lee, A. (2014). A Question of Momentum - Critical Reflections on Individual Options for Surveillance Resistance. Teknokultura: Revista de Cultura Digital y Movimientos Sociales, $11(2), 425-440$.

Linton, A. L. (2015). Politically Engaged and Alienated Youth: Reevaluating 2010 UK Student Protest. En Middaugh, E. y Kirshner, B. (ed.). \#youthaction. Becoming Political in the Digital Era. Charlotte: IAP.

McChesney, R. (2013). Digital Disconnect. How Capitalism is Turning the Internet Against Democarcy. New York: The New Press.

Morozov, E. (2011). The Net Delusion. How not to Liberate the World. London: Allen Lane.

Mosco, V. (2004). The Dygital Sublime. Myth, Power, and Cyberspace. Cambridge: MIT Press.

Navarro, V. (2016). Las ignoradas causas de la enorme crisis que estamos viviendo. Disponible en:

$<$ http://www.vnavarro.org/?p=13150\#more-13150> [Consultado el 12/04/16]

NegRi, A. (1993). El poder constituyente: Ensayo sobre las alternativas de la modernidad. Madrid: Libertarias Prodhufi.

Pennisi, A. (2012). “Entrevista a Toni Negri”. Diario La Nación, 2 de noviembre. Disponible en:

$<$ http://www.lanacion.com.ar/1522453-toni-negri-es-necesario-volver-a-las-palabras-quesignifican-algo $>$ [Consultado el 23/09/14]

Rodríguez, R. y Martínez, F. (2016). Poder e Internet. Un análisis crítico de la Red. Madrid: Cátedra.

Rodríguez, R. (2005). Ciudadanos soberanos. Participación y democracia directa. Córdoba: Almuzara.

Roper, B. S. (2013). The History of Democracy. A Marxist Interpretation. London: Pluto.

Segura VÁzquez, A. (2014). El pastor, el doctor y el Big Data. Teknokultura: Revista de Cultura Digital y Movimientos Sociales , 11 (2), 243-257.

Sherry, D. (2010). Occupy! A Short History of Workers' Occupations. London: Bookmarks Publications.

Soriano, R. (2014). Democracia vergonzante. Males y remedios para una democracia obsoleta. Málaga: Última Línea.

SubiRATs, S. (2015). ¿Desbordar el “dentro”-“fuera”? Teknokultura: Revista de Cultura Digital y Movimientos Sociales . 12 (1), 161-168. 
TAYlor, A. et al. (2011). Occupy. Scenes from Occupied America. New York: Verso.

TeJERINA, B., et al. (2013). "From indignation to occupation: A new wave of global mobilization". "Current Sociology Monograph", 2 61(4).

TejerinA, B. (2010). La sociedad imaginada. Movimientos sociales y cambio cultural en España. Madrid: Editorial Trotta.

Toret, J. (2015). Una mirada tecnopolítica al primer año de Podemos Seis hipótesis. Teknokultura: Revista de Cultura Digital y Movimientos Sociales , 12 (1), 121-135.

WInNer, L. (2014). Facing the Plague. Economic and Political Inequality. Teknokultura: Revista de Cultura Digital y Movimientos Sociales, 11(3), 507-527.

Young, I. M. (1990). Imparcialidad y lo cívico público. Algunas implicaciones de la crítica feminista a la teoría moral y política, en Benhabib, S. y Cornella D. (eds.) Teoría feminista y teoría crítica. Valencia: Alfons El Magnànim. 